\title{
Generalization of the Neupert effect over the solar flare plasma cooling
}

\author{
Arun Kumar Awasthi ${ }^{1, *}$ and Rajmal Jain ${ }^{1}$ \\ ${ }^{1}$ Physical Research Laboratory, India \\ *email: awasthi@prl.res.in
}

\begin{abstract}
We investigate $10 \mathrm{M}$-class flares observed by the SOXS mission to study the influence of the solar flare plasma cooling on the Neupert effect. We study the temporal evolution of 1s cadence X-ray emission in 7-10 keV and 10-30 keV representing the SXR and HXR emission respectively. We model the cooling as a function of time by the ratio of time-derivative of SXR with the HXR flux. We report that the ratio is exponentially decaying in rise phase of the flare, which, however, saturates after the impulsive phase. We estimate the cooling time scale in the rise phase for the flares and found to be varying between 39 and $525 \mathrm{~s}$.
\end{abstract}

Keywords. Sun: flares, X-ray: flares, conduction, radiation

\section{Introduction}

The standard solar flare model explains energy stored in coronal magnetic fields liberated via the process of magnetic reconnection. This energy is used in both heating the local plasma and accelerating charged particles. These particles stream along reconnected field lines toward the dense chromosphere where hard X-ray emission (HXR hereafter) is produced and chromosphere starts evaporation producing soft X-ray (SXR hereafter) emission. Therefore, the rate of change of energy deposited by the accelerated electrons must be reflected into the energy released in the form of SXR, also known as Neupert effect (Dennis and Zarro,1993). However, the plasma producing SXR cools simultaneously by conduction (Jain et al. 2011) in the rise phase and by cooling in the decay phase (Culhane et al., 1970). Therefore, Neupert effect is strictly only expected for the asymptotic limit of very long cooling times (Holman et al., 2011). Ning et al., (2012) have shown that the radiative and conductive losses added to SXR show very good correlation with the HXR. Therefore, in this paper, we explore the posiblity of generalizing neupert effect by adding the effects of cooling.

\section{Data Analysis, Results \& Discussion}

The relation between the evolution of the soft X-ray flux and hard X-ray flux can be modeled with an empirical cooling time (Aschwanden, 2005) as

$$
\frac{d F_{s x r}}{d t} \propto F_{h x r} e^{-t / \tau_{c o o l}}
$$

We study this relation in 10 GOES M-Class flares observed by Solar X-ray Spectrometer experiment (Jain et al. 2005) (cf. Table 1).

In addition to the usual $3 \mathrm{sec}$ spectral and temporal cadence observations, SOXS also provides count flux in 5 energy-bands viz. 6-7, 7-10, 10-20, 20-30, 30-56 with superb time cadence of $1 \mathrm{~s}$. We employ photon flux in 7-10 and (10-20 + 20-30) keV energy bands as SXR and HXR flux respectively. Figure-1- Left top \& bottom panels show the intensity profile of SXR and HXR respectively for the flare event of 19-Nov-03. Figure-1- Right 
Table 1. The observable quantities and flare plasma parameters for the $10 \mathrm{M}$-class flare events

\begin{tabular}{|c|c|c|c|c|c|}
\hline Date & Start Time (UT) & Peak Time (UT) & Peak Intensity $^{1}$ & GOES Class & Temperature (MK) \\
\hline 19-11-03 & $03: 58$ & 04:02 & 3.07 & M1.7 & 26.38 \\
\hline $07-01-04$ & B $03: 55$ & 04:00 & 6.50 & M4.5 & 21.01 \\
\hline 19-01-04 & $04: 35$ & $04: 54$ & 2.45 & M1.0 & 25.64 \\
\hline $25-04-04$ & $05: 28$ & $05: 36$ & 5.80 & M2.3 & 25.88 \\
\hline $14-08-04$ & $04: 13$ & $04: 14$ & 8.80 & M2.4 & 21.63 \\
\hline $31-10-04$ & $05: 26$ & $05: 31$ & 6.04 & M2.3 & 26.48 \\
\hline $03-06-05$ & 04:06 & $04: 10$ & 1.70 & M1.3 & 16.19 \\
\hline $03-08-05$ & $04: 57$ & $05: 05$ & 5.40 & M3.4 & 22.25 \\
\hline $12-09-05$ & $04: 31$ & $05: 00$ & 1.48 & M1.5 & 19.13 \\
\hline $17-09-05$ & 06:01 & $06: 05$ & 14.5 & M9.8 & 20.93 \\
\hline
\end{tabular}

${ }^{1}$ Peak intensity in units of Photons-s ${ }^{-1}-\mathrm{cm}^{-2}-\mathrm{keV}^{-1}$ in $7-10 \mathrm{keV}$ energy band.
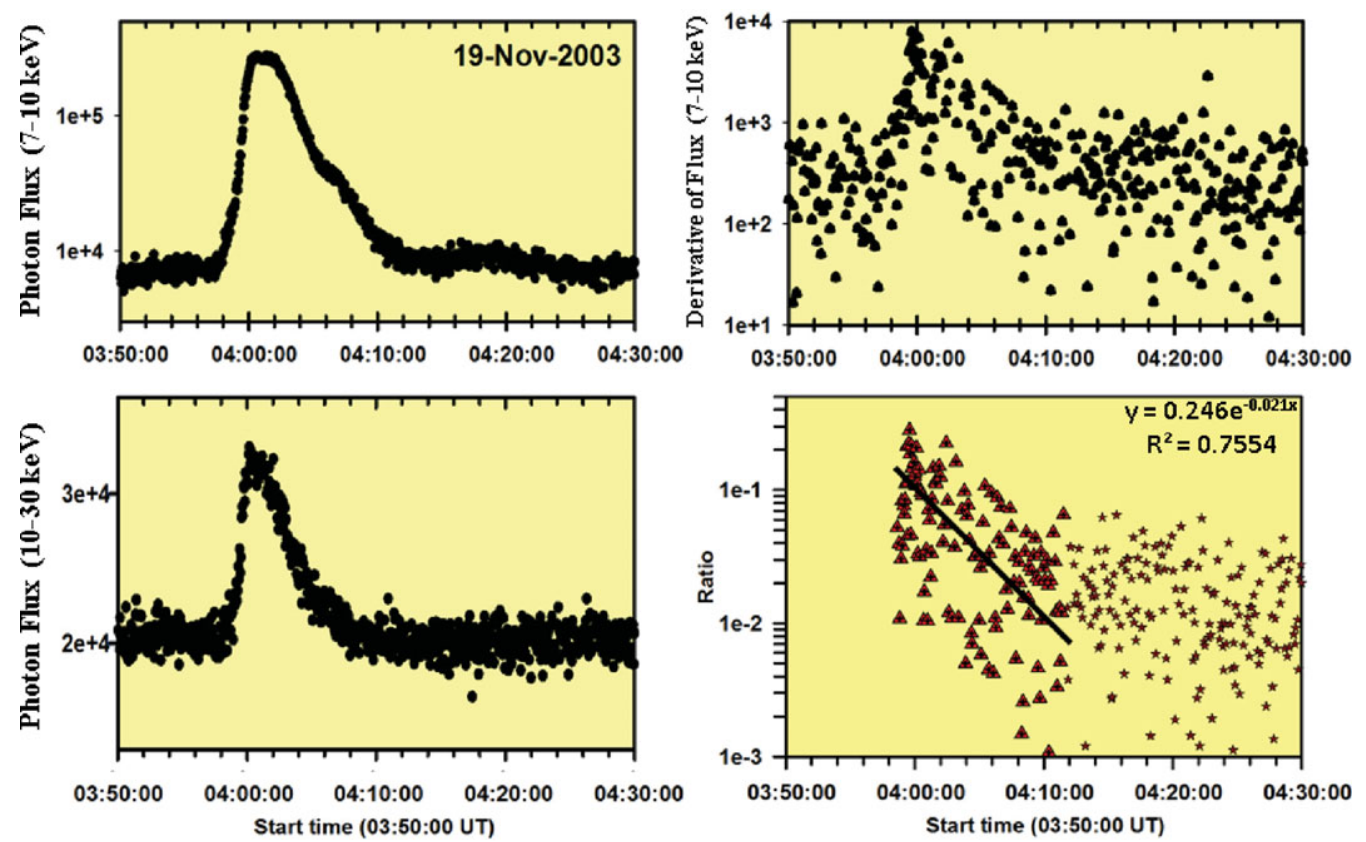

Figure 1. Left top $\mathscr{E}$ bottom panels- Intensity profile of SXR and HXR respectively for the flare event of 19-Nov-03. Right top $\&$ bottom panels- The time derivative of SXR and the ratio of time derivative of flux SXR to flux HXR. Color version is available in online issue.

top \& bottom panels show the time derivative of SXR and the ratio of time derivative of flux SXR to flux HXR.

We fit the time evolution of the ratio by an exponentially decaying function in the rising phase and estimated cooling scale to be $47 \mathrm{~s}$. We estimate this time scale for all flares and found to be varying between 39-525s. Due to its occurrence in rising phase, this scale may be attributed to conduction cooling time scale.

\section{References}

Dennis, B. R. \& Zarro, D. M. 1993, Solar Phys., 146, 177

Culhane, J. L., Vesecky, J. F., \& Phillips, K. J. H. 1970, Solar Phys., 15, 394

Holman, G. D., Aschwanden, M. J., Aurass, H., et al. 2011, Space Sci. Revs, 159, 107

Ning, Z. \& Li, D. 2012, ApESSS, 338, 15

Jain, R., Awasthi, A. K., Rajpurohit, A. S., \& Aschwanden, M. J. 2011, Solar Phys., 270, 137

Achwanden, M. J, 2005, The Physics of Solar Corona: problems and solutions

Jain, R., Dave, H., Shah, A. B., et al. 2005, Solar Phys., 227, 89 